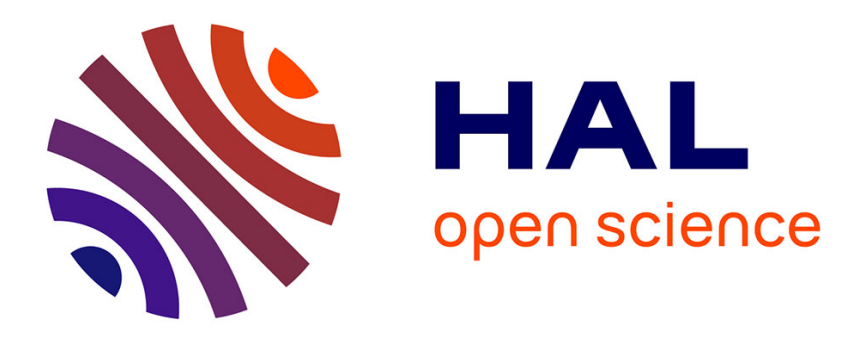

\title{
Inversion of a new V-line Radon transform and its numerical analysis
}

\author{
M.K. Nguyen, Rémi Regnier
}

\section{To cite this version:}

M.K. Nguyen, Rémi Regnier. Inversion of a new V-line Radon transform and its numerical analysis. ICNAAM 2010: International Conference of Numerical Analysis and Applied Mathematics, Sep 2010, Rhodes, Greece. hal-00527128

\section{HAL Id: hal-00527128 \\ https://hal.science/hal-00527128}

Submitted on 18 Oct 2010

HAL is a multi-disciplinary open access archive for the deposit and dissemination of scientific research documents, whether they are published or not. The documents may come from teaching and research institutions in France or abroad, or from public or private research centers.
L'archive ouverte pluridisciplinaire HAL, est destinée au dépôt et à la diffusion de documents scientifiques de niveau recherche, publiés ou non, émanant des établissements d'enseignement et de recherche français ou étrangers, des laboratoires publics ou privés. 


\title{
Inversion of a new V-line Radon transform and its numerical analysis
}

\author{
M. K. Nguyen $\ddagger$ and R. Régnier $\ddagger$ \\ ‡ Laboratoire Equipes Traitement de l'Information et Systèmes, \\ (CNRS UMR 8051/ENSEA/Université de Cergy-Pontoise), \\ 2 av. Adolphe Chauvin, 95302 Cergy-Pontoise, France.
}

\begin{abstract}
A new Radon transform defined on a discontinuous curve formed by a pair of half-lines forming a letter $\mathrm{V}$ is defined and studied. We establish its analytic inverse formula, its related filtered back-projection reconstruction procedure and its numerical analysis. These theoretical results allow the reconstruction of two-dimensional images of a radiating object from its Compton scattered rays measured on a one-dimensional collimated camera. Numerical simulations results illustrate the performance of the new imaging process.
\end{abstract}

\section{Introduction}

The Radon transform of a function $f(x, y)$ [1] has been defined as the integral of $f(x, y)$ on a family of curves in $\mathbb{R}^{2}$, such as straight lines, circles intersecting a fixed point, or more general classes of curves $[2,3]$. It was $\mathrm{R}$ Basko who, in an attempt to model image formation in the so-called one-dimensional Compton camera, introduced a Radon transform on a pair of half-lines forming a V-letter [4]. The axis of his V-line swings around a point of the plane such that its vertex lies on a line with a variable opening angle between the two halflines. Here we consider the class of V-line Radon transforms with fixed $O y$-axis direction and vertex on the $O x$-axis. Such Radon transforms may be of theoretical interest in integral geometry in the sense of Gel'fand [5]. An exact inverse formula will be established and its back-projection form derived. As a possible application we perform numerical simulations on the reconstruction of $f(x, y)$. This may be of use as a new imaging modality in nuclear medicine or in non-destructive testing [7].

\section{The V-line Radon transform}

\subsection{Review of the standard Radon transform}

The standard Radon transform maps a $L^{1}\left(\mathbb{R}^{2}\right)$-function $f(x, y)$ onto $\mathbb{R} f(p, \phi)$, according to

$$
\mathbb{R} f(p, \phi)=\int_{\mathbb{R}^{2}} d x d y f(x, y) \delta(p-x \cos \phi-y \sin \phi),
$$

in which $(p, \phi)$ are the parameters of the straight line: $\phi$ the angle of the unit normal vector $\mathbf{n}$ of the line with the axis $O x$ and $p$ the distance from the polar coordinate system origin. There exists an explicit inverse formula, see e.g. [6]

$$
f(x, y)=-\frac{1}{2 \pi^{2}} \text { P.V. } \int_{0}^{\pi} d \phi \int_{\mathbb{R}} d p \frac{\mathbb{R} f(p, \phi)}{(p-x \cos \phi-y \sin \phi)^{2}},
$$


where P.V. denotes the Cauchy principal value of the $p$-integral. This inverse formula can be recast as a filtered back-projection formula, after reordering the integrations as follows

$$
f(x, y)=\int_{\mathbb{R}^{2}} d \mathbf{k}|\mathbf{k}| e^{2 i \pi \mathbf{k} \cdot \mathbf{r}}\left\{\int_{\mathbb{R}^{2}} d \mathbf{r}^{\prime} e^{-2 i \pi\left(\mathbf{r}^{\prime} \cdot \mathbf{k}\right)} \int_{0}^{\pi} d \phi \int_{\mathbb{R}} d p \mathbb{R} f(p, \phi) \delta\left(p-\left(\mathbf{n} \cdot \mathbf{r}^{\prime}\right)\right)\right\} .
$$

\subsection{Definition of the V-line Radon transform}

Consider a pair of half-lines meeting at a point $\mathbf{M}$ of abscissa $\xi$ on the $O x$-axis, each of which making an angle $\omega(0<\omega<\pi / 2)$ with the $O y$-axis. Let $f(x, y)$ be a $L^{1}\left(\mathbb{R}^{2}\right)$ nonnegative continuous function with compact support in $\left\{\mathbb{R}^{2} \mid y>0\right\}$. We call

$$
\mathbb{V} f(\zeta, \tau)=\int_{0}^{\infty} d r(f(\zeta+r \sin \omega, r \cos \omega)+f(\zeta-r \sin \omega, r \cos \omega)),
$$

the V-line Radon transform of $f$, where $\tau=\tan \omega$.

\subsection{Inversion formula}

To obtain the analytic inversion formula, we show that the relation between the one-variable Fourier transforms of the function and of its V-line Radon transform is, after appropriate change of variables, a simple cosine-Fourier transform, hence

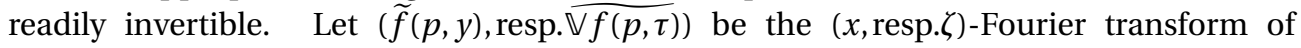
$(f(x, y)$, resp. $\mathbb{V} f(\zeta, \tau))$

$$
f(x, y)=\int_{-\infty}^{\infty} d p e^{2 i \pi p x} \tilde{f}(p, y) \text { resp. } \mathbb{V} f(\zeta, \tau)=\int_{-\infty}^{\infty} d \zeta e^{2 i \pi p \zeta} \overline{\mathbb{V f}(p, \tau)} .
$$

Let $z=r \cos \omega$ be a new integration variable. Since $0<\omega<\pi / 2, \cos \omega=\frac{1}{\sqrt{1+\tau^{2}}}>0$ and $z>0$. Then eq. (4) reads

$$
\frac{\overline{\mathbb{f ( p , \tau})}}{2 \sqrt{1+\tau^{2}}}=\int_{0}^{\infty} d z \tilde{f}(p, z) \cos (2 \pi p \tau z)
$$

This is precisely a cosine-Fourier transform. Its inverse can be computed straightforwardly under the form

$$
\widetilde{f}(p,|v / p|)=2|p| \int_{0}^{\infty} d \tau \cos (2 \pi \tau v) \int_{\mathbb{R}} d \zeta e^{-2 i \pi \zeta p}\left(\frac{\mathbb{V} f(\zeta, \tau)}{\sqrt{1+\tau^{2}}}\right) .
$$

Putting $y=|v / p|>0, f(x, y)$ is reconstructed by inverse Fourier transform in $p$ and is expressed as a triple integral on the V-line Radon data

$$
f(x, y)=\int_{-\infty}^{\infty}|p| d p e^{2 i \pi p x} \int_{0}^{\infty} d \tau \frac{2 \cos (2 \pi p \tau y)}{\sqrt{1+\tau^{2}}} \int_{-\infty}^{\infty} d \zeta e^{-2 i \pi \zeta p} \mathbb{V} f(\zeta, \tau),
$$

where we have made use of $\cos (2 \pi \tau \nu)=\cos (2 \pi \tau|\nu|)=\cos (2 \pi \tau y|p|)=\cos (2 \pi \tau y p)$. For $y>0$, explicit intermediate integrations yield the final reconstruction formula :

$$
f(x, y)=-\frac{1}{2 \pi^{2}} \int_{0}^{\infty} \frac{d \tau}{\sqrt{1+\tau^{2}}} \mathrm{P.V} .\left(\int_{\mathbb{R}} d \zeta\left(\frac{\mathbb{V} f(\zeta, \tau)}{(x+\tau y-\zeta)^{2}}+\frac{\mathbb{V} f(\zeta, \tau)}{(x-\tau y-\zeta)^{2}}\right)\right) .
$$




\subsection{Filtered back-projection form of inversion}

Equation (9) can be put also under the form of a filtered back-projection as in the case of the standard Radon transform

$$
\begin{gathered}
f(x, y)=\int_{\mathbb{R}^{2}} d \mathbf{k}|\mathbf{k}| e^{2 i \pi \mathbf{k} \cdot \mathbf{r}} \int_{\mathbb{R}^{2}} d \mathbf{r}^{\prime} e^{-2 i \pi\left(\mathbf{r}^{\prime} \cdot \mathbf{k}\right)} \\
\left\{\int_{0}^{\pi / 2} d \omega \int_{\mathbb{R}} d u \mathbb{V} f\left(\frac{u}{\cos \omega}, \frac{\sin \omega}{\cos \omega}\right)\left[\delta\left(u-\left(\mathbf{n} \cdot \mathbf{r}^{\prime}\right)\right)+\delta\left(u-\left(\mathbf{n}^{\prime} \cdot \mathbf{r}^{\prime}\right)\right)\right]\right\} .
\end{gathered}
$$

$\mathbf{n}$ and $\mathbf{n}^{\prime}$ are unit vectors normal to the two branches of the V-line. The term in curly brackets is just the Barrett summation image of the V-line Radon transform but over a half angular range only with the same filtering as in Radon transform.

\section{Numerical simulations}

The V-line Radon transform arises from modeling two-dimensional emission imaging process by Compton scattered gamma rays. The image reconstruction is performed using the filtered back-projection inversion formula (10) of the V-line Radon transform. In figures $(1,2,3)$ we show an original thyroid medical phantom, its V-line Radon transform data and its reconstruction from simulated data. Back-projection on V-lines generates more artifacts than back-projection on straight lines in standard two-dimensional Radon Transform, due to the existence of more spurious line intersections. In order to reduce these artifacts, the Hann filter may be used. Despite these limitations, the small structures in the object are clearly reconstructed. These results illustrate undoubtedly the feasibility of the new imaging modality.

\section{Conclusion}

In this paper, a novel class of Radon transform defined on a discontinuous line having the shape of a V letter is shown to be analytically invertible and the corresponding filtered back-projection inversion procedure is very much akin with that of the standard Radon transform. This formula is then used for numerical simulations of a medical thyroid phantom. The results show clearly the feasibility of reconstruction and suggest applications in many fields such as nuclear medicine or non-destructive material testing.

[1] Radon J 1917 Über die Bestimmung von Funktionnen durch ihre Integralwerte längs gewisser Mannigfaltikeiten Ber.Verh.Sachs.Akad.Wiss. Leipzig-Math.-Natur.Kl.69, 262-277.

[2] Cormack A M 1983 The Radon transform on a family of curves in the plane Proceedings of the American Mathematical Society 83(2), 325-330.

[3] Quinto E T 1994 Radon transforms on curves in the plane Lectures in Applied Mathematics: Tomography, Impedance Imaging and Integral Geometry 30, 231-244.

[4] Basko R, Zeng G L, Gullberg G T 1997 Analytical reconstruction formula for the one-dimensional Compton camera, IEEE Trans. Nucl. Sci. 44(3), 1342-1346.

[5] Gelfand I M 1960 Integral geometry and its relations to the theory of group representation Russian Math. Surveys 15, 143-151.

[6] Barrett H H 1984 The Radon Transform and its Applications in Progress in Optics 21, 219-286, Ed. E. Wolf, North Holland.

[7] Truong T T and Nguyen M K 2008 On a class of generalized Radon transform and its role in imaging science, International Journal of Pure and Applied Mathematics, 49(3), 373-380. 


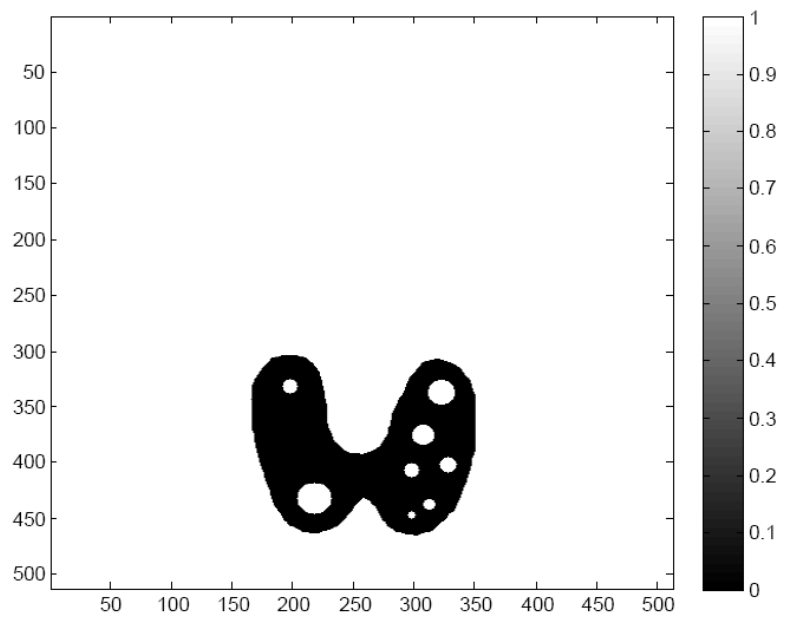

Figure 1. Original thyroid phantom.

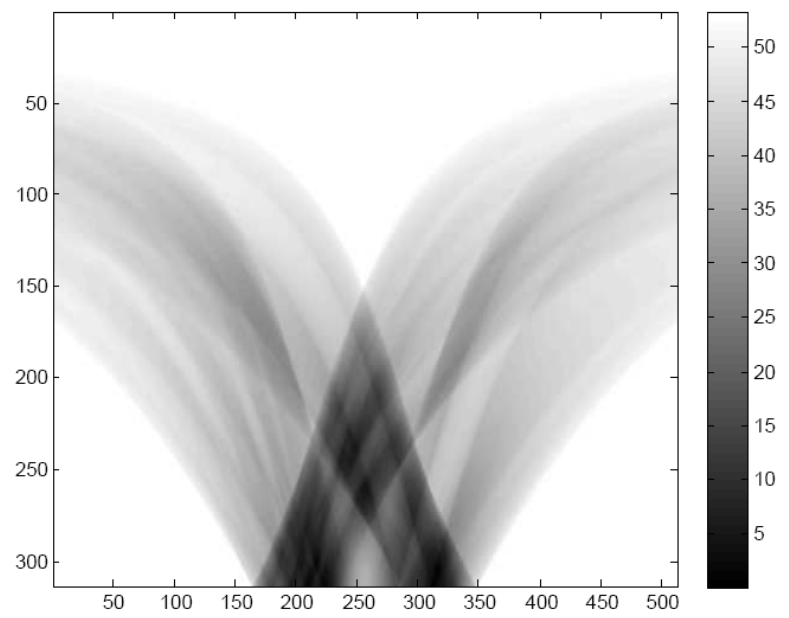

Figure 2. The V-line Radon transform of the thyroid with $d \omega=0.005$ rad.

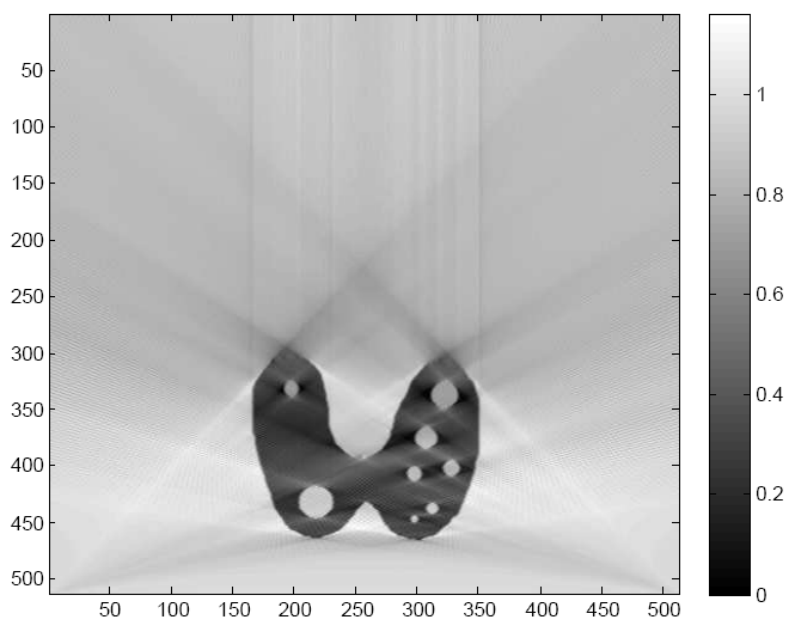

Figure 3. Filtered back-projection reconstruction with ( $d \omega=0.005 \mathrm{rad})$. 